\title{
SVM-Based Decision Fusion Model for Detecting Concepts in Films
}

\author{
P. Muneesawang \\ Department of Electrical and Computer Engineering \\ Naresuan University \\ Phisanulok, Thailand 65000 \\ paisarnmu@nu.ac.th
}

\author{
L. Guan \\ Depart. of Electrical and Computer Engineering \\ Ryerson University \\ Toronto, Canada M5B 2E8 \\ lguan@ee.ryerson.ca
}

\begin{abstract}
This paper studies a support vector machine (SVM) to obtain a decision fusion algorithm for detection of semantic concepts in videos, and its application to Films database. Given a movie clip, its spatio-temporal information is captured by audiovisual features. These are then independently inputted to the corresponding matching experts whose outputs are fused at the decision stage by the SVM classifier. Based on our simulation results, this fusion method can attain very high recognition accuracy for detection of various concepts from a collection of Hollywood movies. It requires a very small set of training samples from a large database.
\end{abstract}

Keywords-SVM decision fusion, semantic concept detection, movie retrieval, audiovisual indexing

\section{INTRODUCTION}

The application of multimodality signal processing has been demonstrated to help solve challenge problems in multimedia databases, such as story segmentation, concept detection, retrieval, and topic clustering. Much of the previous works have been focused mainly on news videos and sports domain applications. A key challenge here is to integrate the different sources of evidence from many multimedia features into indexing that helps system effectively find what user seeks. Upon the nature of news videos, the text information from speech transcription and closed caption can be exploited. This is fused together with audio-visual features showing promising performance in processing for broadcast news video domain. Some of the recent research works on this topic are discussed in [1]. For sport videos, although they are of the same type, sport videos represent different genres and require specific approaches for detecting useful attributes. These include the following techniques that detect events (e.g., goals and penalty) in baseball [2], cricket [3], and tennis [4], which typically yield successful results within the targeted domain. In addition, the detection task requires a more 'generic approach' that is common to all genres of sports (e.g., [5]-[7]).

The methods discussed so far are specified either to news and sports videos, and relatively little prior works addressed the related problems for movie domain application. Central to all these works are complex algorithms, performing standalone modeling of specific events, based on intrinsically critical characteristic features, which tend to be particular to each video type. Their effectiveness is somewhat diluted by their inherent inapplicability to other video genres. In addition, a more generic, genre independent methodology is the more challenging and difficult task. For a given event detection task, it is unfeasible to consider that there exists a unique solution that will operate successfully across all genres of video. We propose in this paper to address the problems for movie domain application where only few previous works have been done. Rassheed et al [8] has worked on the classification of movies into broad categories: Comedies, Action, Dramas, or Horror films. Inspired by cinematic principles, computable features such as motion content and lighting key are applied to map a movie into semantic classes.

Combining different modalities allows to alleviate problems intrinsic to single modalities, and a fusion algorithm, which combines the different modalities, is a very critical part of the recognition system [9]. In the current work, the audio and visual features are fused by a learning module to characterize concepts. The learning module is implemented by a passive learning process using support vector machine for detection of concepts according to pre-defined classes. The SVM-based decision fusion has been demonstrated in other application domains including cartridge identification [10] as well as person identity verification [11]. We propose an adoption of SVM to obtain fusion algorithm at the decision stage for characterization of concepts. Based on our experimental results the proposed system deploying perception features extracted from audiovisual data, together with the SVM-based decision fusion models offered very high recognition accuracy and required a small set of training data when applied to a large database of Hollywood movies.

\section{SVM-BASED DECISION FUSION}

\section{A. Fusion model}

Figure 1 shows a diagram of the proposed fusion model using SVM as a classifier. The extracted data (audio and visual) are processed by different matching experts: an audio similarity matching expert and a visual similarity matching expert. Each expert, given the extracted data, will delivers a matching score in the range between zero (reject) and one (accept). These scores are not binary decisions. The SVM fusion module will combine the opinions of the different experts and give a binary decision. When combining 2 modules, the fusion algorithm process 2-dimentional vectors whose each component is a matching score in $[0,1]$ delivered by the corresponding modality expert. This paper will address the issue of the fusion model using two types of features. 


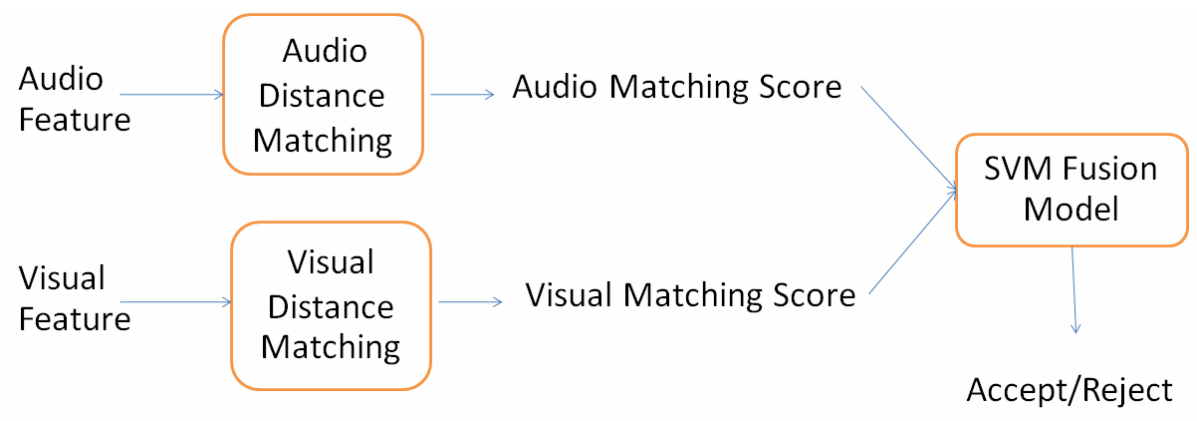

Figure 1: SVM-based decision fusion model

\section{B. Support Vector Machine (SVM)}

A retrieval task may be viewed as a pattern recognition problem when some data items in database are available for training. In contrast to the active learning process [17] where users provide the class labels during retrieval process, the pattern recognition task requires pre-labeled data items for training before recognition stage. In this paper we study a discriminate learning approach, support vector machine (SVM) for leaning audiovisual concepts for video classification. A SVM-based decision fusion technique has been demonstrated for cartridge identification [10] as well as for person identity verification [11].

The SVM approach is considered a good candidate for pattern classification because its high generalization performance. It is based on the principle of structural risk minimization (SRM) that tries to find the best fitting hyperplane that maximizes the generalization capabilities while minimizing misclassification errors. Assume that we have a data set $D$ of $l$ points in an $n$-dimensional space belonging to two different classes +1 and -1 . That is:

$$
\begin{gathered}
D=\left\{\left(\mathbf{x}_{i}, y_{i}\right) \mid i \in\{1, \ldots, l\}\right\}, \\
\mathbf{x}_{i} \in R^{n}, y_{i} \in\{+1,-1\}
\end{gathered}
$$

Then SVMs map the samples from their data space to their label space using a mapping function, $f$ :

$$
\begin{aligned}
f: R^{n} & \rightarrow\{+1,-1\} \\
& \mathbf{x}_{i} \rightarrow y_{i}
\end{aligned}
$$

The decision function is:

$$
f(\mathbf{x})=\operatorname{sign}\left(\sum_{i} \alpha_{i} y_{i} K\left(\mathbf{x}_{i}, \mathbf{x}\right)+b\right)
$$

where $K\left(\mathbf{x}_{i}, \mathbf{x}\right)$ is a positive definite symmetric function, $b$ is a bias estimated on the training set, $\alpha_{i}$ are the solution of the following quadratic programming problem [16]:

$$
\min _{\alpha} \frac{1}{2} \boldsymbol{\alpha}^{T} \mathbf{Q} \boldsymbol{\alpha}+\mathbf{e}^{T} \boldsymbol{\alpha}
$$

subject to

$$
\begin{gathered}
\mathbf{y}^{T} \boldsymbol{\alpha}=0 \\
0 \leq \alpha_{i} \leq C, i=1, \ldots, l,
\end{gathered}
$$

where $\mathbf{e}$ is the vector of all ones, $C>0$ is the upper bound, $\mathbf{Q}$ is an $l$ by $l$ positive semidefinite matrix $Q_{i j} \equiv y_{i} y_{j} K\left(\mathbf{x}_{i}, \mathbf{x}_{j}\right)$. The nature of the decision surface which will separate the data is defined by the kernel functions $K\left(\mathbf{x}_{i}, \mathbf{x}_{j}\right)$. These kernel functions satisfy some constraints in order to be applicable to Mercer's conditions. We use the radial basis function (RBF) in this paper. The RBF is defined as:

$$
K\left(\mathbf{x}_{i}, \mathbf{x}_{j}\right)=\exp \left(-\gamma\left\|\mathbf{x}_{i}-\mathbf{x}_{j}\right\|^{2}\right), \gamma>0
$$

where $\gamma$ is the kernel parameter.

\section{Feature Extraction}

In order to obtain audiovisual features inputted to the fusion module discussed in Figure 1, we employ an adaptive video indexing technique demonstrated in [12] for visual characterization. In addition, we adopt the audio features based on Laplacian mixture model (LMM) [13]. These are summarized as follows:

Visual Feature: The AVI technique is in the same spirit as the popular vector quantization algorithm. A video is a collection of frames each of which is considered as a vector point. All vectors in a video clips are mapped or quantized to an optimum set of representative vectors. We applied a color histogram (using HSV color space) to extract color feature from frames in a video clip. A number of color histogram vectors randomly selected from all video clips in the database were used for training by the competitive learning algorithm [12]. This produced a set of optimum visual templates with size of 2,000. These templates were used by the mapping process for obtaining weight vector for visual feature.

Audio Feature: A wavelet transform with 9-level decompositions was applied to audio signal from each video clip. The 'detail' coefficients in each sub-band were then characterized by LMM, and the model parameters were used to obtain 29-dimensional feature vector. By this way, as feature components represent different physical quantities and have different dynamic ranges, Gaussian normalization was employed to convert the component feature to $[-1,1]$. 


\section{SVM-based Decision Fusion algorithm}

The application of SVM-based fusion algorithm for concept detection is summarized as follows:

\section{SVM-based Decision Fusion Procedure}

Training stage:

For a given concept, select a representative video clip and obtain its corresponding features, $\mathbf{v}_{q}^{(V)}$ and $\mathbf{v}_{q}^{(A)}$

For $t=1,2, \ldots, T$,

Calculate $D^{(V)}[t]=1-\sqrt{\sum_{i=0}^{M-1}\left(v_{q}^{(V)}[i]-v_{t}^{(V)}[i]\right)^{2}}$

Calculate $D^{(A)}[t]=\frac{\mathbf{v}_{q}^{(A)} \cdot \mathbf{v}_{t}^{(A)}}{\left\|\mathbf{v}_{q}^{(A)}\right\|\left\|\mathbf{v}_{t}^{(A)}\right\|}$

Obtain training vector, $\mathbf{x}=\left\lfloor D^{(V)}[t] D^{(A)}[t]\right]$, and its

corresponding label, $y \in\{-1,+1\}$

Randomly select data from $\left(\mathbf{x}_{i} y_{i}\right), i=1, \ldots, T$ to obtain training set, $D=\left\{\left(\mathbf{x}_{i}, y_{i}\right) \mid i \in\{1, \ldots, l\}\right\}$, where $l \ll T$

Train the SVM models

Recognition state:

Use the resulting mapping function, $f$ for prediction, $\mathbf{x}_{i} \rightarrow y_{i}$ for $i=1, \ldots, T$

Measure classification accuracy

NOTE - List of Terms:

$\mathbf{v}_{q}^{(V)}$ and $\mathbf{v}_{q}^{(A)}$ are the visual feature vector and audio feature vector, respectively.

$D^{(V)}[t]$ and $D^{(A)}[t]$ are the $t$-th matching scores obtained by the visual feature and audio feature, respectively.

$T$ is the total number of data items in the database.

\section{EXPERIMENTAL RESULTS}

The experimental results obtained in this section were conducted on a database consists of 24 full-length and mostly recent mainstream Hollywood movies chosen to represent the more popular films, music videos, and commercial. This translates into 6,000 clips, each of which contains one to three shots.

In Figure 2, five query concepts were utilized to obtain the results. These concepts include fighting, ship crashing, love scene, music video, and dancing party. We applied SVM-based decision fusion models described in Section II(D) for classification of concepts from video database. For each of the five concepts, we obtained the ground true classes by manually classify all video clips in the database. These classes were used for measuring classification performance of the SVM models. Table 1 shows detail information of the data set used in the experiment. For each concept, SVM models were trained by a training set whose size of 100-300 samples according to the type of concepts. The size of this training set was approximately less than $2 \%$ of all video clips used for testing.

The reason of using small set of samples for training is the following. Although the goal here is to classify all video clips in the database into two classes: relevant (positive) and nonrelevant (negative) classes, in the current work SVM classifiers were utilized differently from other classification problems. For this video classification problem, we observed that within a 'positive class' each video clip is similar to other video clips subjected to a specific concept. In comparison, all videos within a 'negative class' were not necessary similar to each others. We observed that the characteristic of relevancy of positive samples is important for the classifier to learn. In contrast, the relationship between samples in the negative class is difficult to characterize and classify into one class. These characteristics imply that positive samples are more important than the negative samples in order to achieve a better training for the classifier. In the experiments, we included in the training set a relatively high percentage of positive samples and a low percentage of negative samples, as shown in Table 1.

In order to measure the performance of the SVM models for concept classification, we used three following criterions: classification accuracy, false positive rate, and false negative rate. The classification accuracy is used to measure the percentage of correct/incorrect classification [14], whereas the other two metrics are defied as followed [15]:

$$
\begin{aligned}
& \text { false positive rate }=\frac{N_{f p}}{N_{n}} \\
& \text { false positive rate }=\frac{N_{f n}}{N_{p}}
\end{aligned}
$$

where $N_{f p}$ is the number of false positives, $N_{n}$ is the number of negative instances, $N_{f n}$ is the number of false negatives, and $N_{p}$ is the number of positive instances.

The false positive rate is the proportion of negative instances that were erroneously reported as being positive, and the false negative rate is the proportion of positive instances that were erroneously reported as negative.

Table 2 shows the experimental results obtained by the SVM-based decision fusion model for classification of video clips. It can be observed that the model achieved a very high accuracy at more than $91 \%$ averaged. It should be noted that this is not a rare result. The number of negative samples is much more than the desired (positive) items within a given concept; the models can correctly classified most of the negative items, and thus it can achieve a high average. 


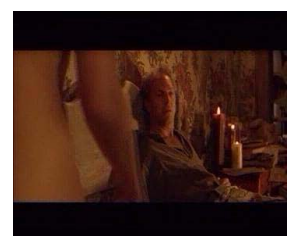

LS1

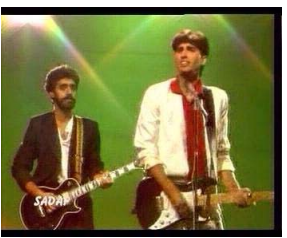

MS1

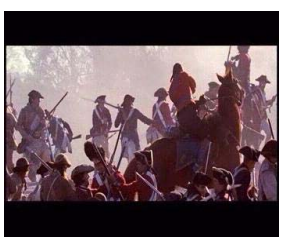

F1

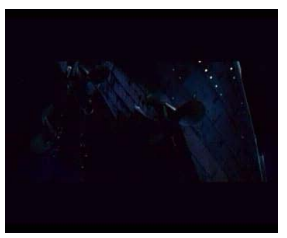

SC1

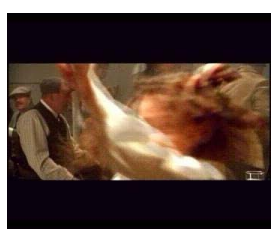

DP1

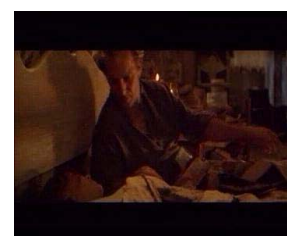

LS2

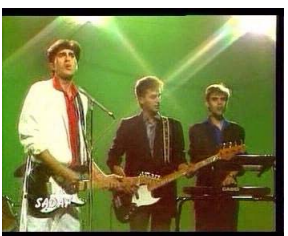

MS2

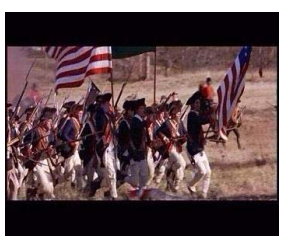

F2

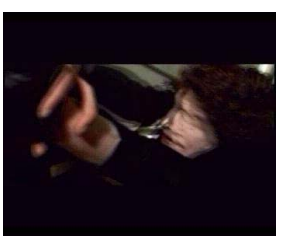

SC2

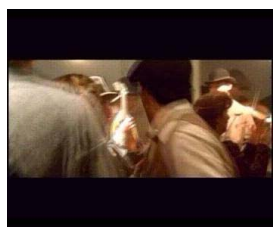

DP2

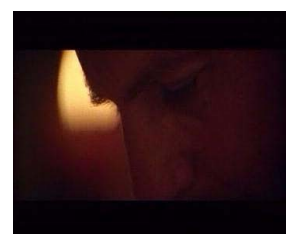

LS3

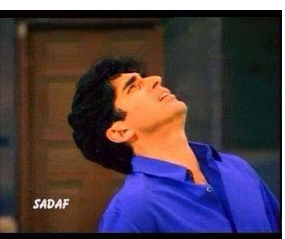

MS3

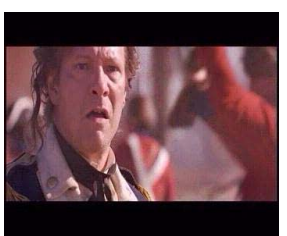

F3

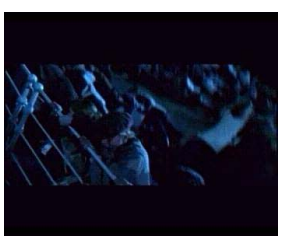

SC3

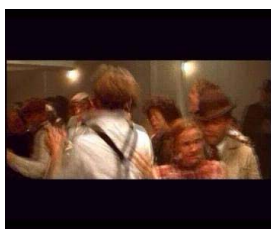

DP3

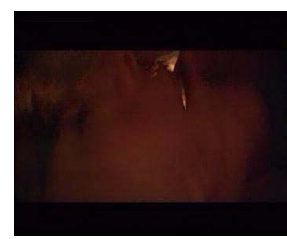

LS4

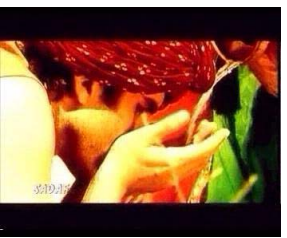

MS4

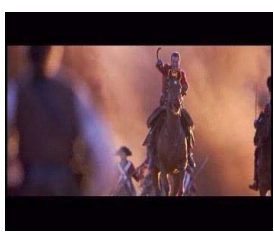

F4

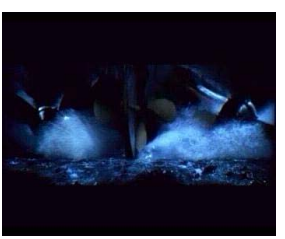

SC4

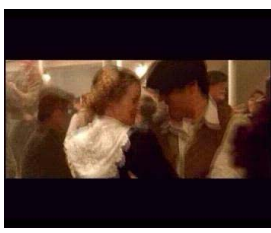

DP4

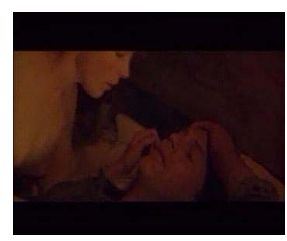

LS5

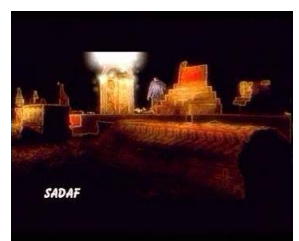

MS5

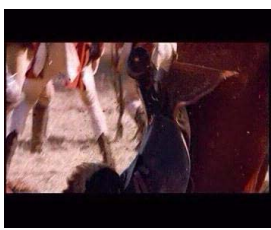

F5

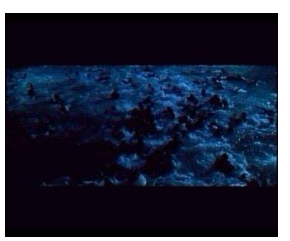

SC5

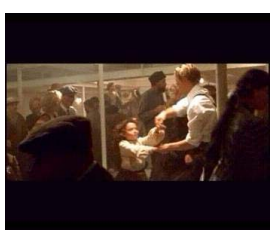

DP5

Figure 2: Sample video clips (each of which is shown by its corresponding key frame) specified to the following concepts: Love Scene (LS1-LS5), Music Video (MS1-MS5), Fighting (F1-F5), Ship Crashing (FC1-FC5), and Dancing Party (DP1-DP5)

An interesting observation may be at the false negative rate since it indicates the percentage of positive items that were correctly detected. The model has the highest false negative rate at $26.87 \%$ for classification of the 'Ship Crashing Concept'. This shows, however, that more than $73 \%$ of all relevant videos were correctly detected. The model can attain the lowest false negative rate of $0 \%$ and $2 \%$ for the detection of 'Music Video' and 'Dancing Party', respectively. For such concepts, we observed that audio features extracted from video clips contributed highly to the effectiveness of the classifier. Furthermore, the consistence visual scene in the video clips representing the 'Dancing Party' concept, together with the music signals used for obtaining audiovisual fusion model enabled the classifier to achieve closely $100 \%$ accuracy.

\section{IV) CONCLUSION}

Detection of concepts from videos is very important and plays an important role for multimedia database management tasks such as retrieval, searching, and topic classification. We have presented in this paper a methodology for detection of concepts from movie clips using a machine learning approach, a SVM-based decision fusion that fuses audiovisual features to characterize the desired concepts. This model requires little training samples in order to achieve its generalization capabilities. This confirms by our simulation results of learning various concepts from a large Hollywood movie database. 
TABLE I. GROUND TRUE AND TRAINING/TESTING DATA USED FOR CONCEPT CLASSIFICATION VIA SVM-BASED DECISION FUSION MODEL.

\begin{tabular}{|c|c|c|c|}
\hline & $\begin{array}{c}\text { Number of } \\
\text { Positive } \\
\text { Data out of } \\
\mathbf{6 , 0 0 0}\end{array}$ & $\begin{array}{c}\text { Number of } \\
\text { Training samples } \\
\text { (positive and } \\
\text { negative) }\end{array}$ & $\begin{array}{c}\text { Number of } \\
\text { Testing } \\
\text { samples }\end{array}$ \\
\hline Love Scene & 66 & $(22,78)=100$ & 6,000 \\
\hline Music video & 41 & $(13,87)=100$ & 6,000 \\
\hline Fighting & 413 & $(137,163)=300$ & 6,000 \\
\hline $\begin{array}{c}\text { Ship } \\
\text { Crashing }\end{array}$ & 201 & $(67,134)=250$ & 6,000 \\
\hline $\begin{array}{c}\text { Dancing } \\
\text { Party }\end{array}$ & 48 & $(16,84)=100$ & 6,000 \\
\hline
\end{tabular}

TABLE II. RECOGNITION RATE OBTAINED BY SVM BASED DECISION FUSION MODEL

\begin{tabular}{|c|c|c|c|}
\hline & $\begin{array}{c}\text { Accuracy } \\
\mathbf{( \% )}\end{array}$ & $\begin{array}{c}\text { False Positive } \\
\text { Rate (\%) }\end{array}$ & $\begin{array}{c}\text { False } \\
\text { Negative } \\
\text { Rate (\%) }\end{array}$ \\
\hline Love Scene & 90.97 & 8.91 & 19.70 \\
\hline Music video & 91.03 & 9.03 & Zero \\
\hline Fighting & 84.68 & 25.65 & 14.55 \\
\hline $\begin{array}{c}\text { Ship } \\
\text { Crashing }\end{array}$ & 91.81 & 7.54 & 26.87 \\
\hline $\begin{array}{c}\text { Dancing } \\
\text { Party }\end{array}$ & 99.68 & 0.30 & 2.08 \\
\hline $\begin{array}{c}\text { Average } \\
\text { average }\end{array}$ & $\mathbf{9 1 . 6 3}$ & $\mathbf{1 0 . 2 9}$ & $\mathbf{1 2 . 6 4}$ \\
\hline
\end{tabular}

\section{REFERENCES}

[1] S.-F. Chang, R. Manmatha, and T.-S. Chua, "Combining text and audiovisual features in video indexing," IEEE International Conference on Acoustics, Speech, and Signal Processing, Philadelphia, 2005, vol. 5, pp. 1005-1008.

[2] S. Nepal, U. Srinivasan, and G. Reynolds, "Automatic detecton of goal segments in basketball videos," Proceeding of AC Multimedia, 2001, pp. 261-269.
[3] M. Lazarescu, S. Venkatesh, and G. West, "On the automatic indexing of cricket using camera motion parameters," Proceeding of IEEE International conference on Multimedia and Expo, 2002, pp. 809-813.

[4] G. Sudhir, J.C.M. Lee, and A.K. Jain: "Automatic classification of tennis video for high-level content-based retrieval", Proceedings of the IEEE International Workshop on Content-based Access of Image and Video Database, 1998, pp. 81-90.

[5] D. A. Sadlier and N. E. O'Connor, "Event detection in fild sports video using audio-visual featues and a support vector machine," IEEE Transaction on Circuit and Systems for Video Technology, vol. 15, no. 10, October, 2005, pp. 1225-1233.

[6] A. Hanjalic, "Gerneric approach to highlights extraction from a sport video," Proceeding of IEEE International Conference on Image Processing, 2003, pp. 1-4.

[7] C. Wu, Y.-F. Ma, H.-J. Zhang, and Y.-Z. Zhong, "Events recogniton by semantic infrence for sports video," Proceeding of IEEE International Conference on Multimedia and Expo, Lausanne, 2002, pp. 805-808.

[8] Z. Rasheed, Y. Sheikh, and M. Shah, "On the use of computable features for film classification," IEEE Trans. Circuits Syst. Video Techn., 2005, vol. 15, pp. 52-64.

[9] S. Ben-Yacoub, "Multimodal data fusion for person authenticatin using SVM," International Conference on Audio-Video Based Biometric Person Authentication, Washington, D.C., 1999, pp. 25-30.

[10] J. Zhou, L.-P. Xin, and G. Rong, "Decision fusion based cartridge identification using support vector machine," IEEE Int. Conference on Systems, Man, and Cybernetics, TN USA, pp. 2873-2877, 2000.

[11] S. Ben-Yacoub, Y. Abdeljaoued, and E. Maoraz, "Fusion of face and speech data for person identity verification," IEEE Transactions on Neural Networks, vol. 10, no. 5, 1999, pp. 1065-1074.

[12] P. Muneesawang and L. Guan, "Adaptive video indexing and automatic/semi-automatic relevance feedback," IEEE Transactions on Circuits and Systems for Video Technology, 2005, vol. 15, Issue 8, pp. 1032- 1046.

[13] P. Muneesawang, T. Amin and L. Guan, "Audio visual cues for video indexing and retrieval," Advances in Multimedia Information Processing - PCM 2004, Springer Berlin/Heidelberg, pp. 642-649.

[14] C.-C. Chang and C.-J. Lin. Training w-support vector classifiers: Theory and algorithms, Neural Computation, 13(9), 2001, pp. 2119-2147.

[15] Wikipedia, "Type I and type II errors," online: http://en.wikipedia.org/wiki/Type I and type II errors, 2007.

[16] C. Cortes and V. Vapnik, "Support-vector network," Machine Learning, 1995, pp 273-297.

[17] K.-H. Yap and K. Wu, "Fuzzy relevance feedback in content-based image retrieval systems using radial basis function network," IEEE International Conference on Multimedia and Expo, 2005, pp. 4-8. 